\title{
X-ray Scattering Studies of Correlated Polarons in $\mathrm{La}_{0.7} \mathrm{Ca}_{0.3} \mathrm{MnO}_{3}$
}

\author{
C.S. Nelson, ${ }^{1}$ M. v. Zimmermann, ${ }^{1}$ J.P. Hill, ${ }^{1}$ Doon Gibbs, ${ }^{1}$ V. Kiryukhin, ${ }^{2}$ \\ T.Y. Koo, ${ }^{2,3}$ and S-W. Cheong ${ }^{2,3}$ \\ 'Department of Physics, Brookhaven National Laboratory, Upton, NY \\ 11973-5000 \\ ${ }^{2}$ Department of Physics and Astronomy, Rutgers University, Piscataway, \\ NJ 08854 \\ ${ }^{3}$ Bell Laboratories, Lucent Technologies, Murray Hill, NJ 07974
}

\begin{abstract}
We report $x$-ray scattering studies of diffuse peaks in the paramagnetic insulating phase of $\mathrm{La}_{0.7} \mathrm{Ca}_{0.3} \mathrm{MnO}_{3}$. The peaks occur at a $(0.500) /(00.50)$-type wavevector, and are found to disappear abruptly upon cooling through the transition into the ferromagnetic metallic phase. Based on the wavevector and measured correlation length of 1-2 lattice constants, the peaks are found to be consistent with the formation of CE-type bipolarons, and their collapse at $T_{c}$ is attributed to the energy gain associated with itineracy in the ferromagnetic state.
\end{abstract}

Electron-phonon coupling is a supplementary mechanism added to the double exchange interaction ${ }^{1}$ in order to model the behavior of colossal magnetoresistance (CMR) materials. ${ }^{2,3}$ This coupling is thought to result in the formation of localized electrons with associated lattice distortions-or polarons--in the paramagnetic insulating phase of CMR materials. Early evidence of polarons was observed using transport measurements ${ }^{4,5}$ and local probes; ${ }^{6,7}$ more recently, interest has turned to studies of polaron correlations. On the theoretical front, one model due to Alexandrov and Bratkovsky ${ }^{8}$ involves the formation at high temperatures of immobile pairs of polarons, which are broken apart at $T_{c}$. Evidence of such bipolarons has recently been reported, ${ }^{9,10}$ but additional studies using more direct techniques are warranted.

Since $x$-rays and neutrons are sensitive to both polarons and their correlations through the associated lattice distortions, these two techniques can make a significant contribution to studies of polarons in CMR materials. To date, $x$-ray and neutron scattering have been used to probe the high-ternperature phases of several different CMR systems, ${ }^{11,12,13,14,15}$ and two types of scattering have been reported: diffuse scattering in the tails of Bragg peaks, and well-resolved peaks. These scattering components have been attributed to the presence of individual polarons and correlated polarons, respectively, and both are observed to disappear as the samples are cooled through their transitions into low temperature phases. The goal of the present study was to use measurements of both types of 
scattering in order to learn about the role played by electron-phonon coupling in the $\mathrm{CMR}$ material $\mathrm{La}_{0.7} \mathrm{Ca}_{0.3} \mathrm{MnO}_{3}$. The work reported here was carried out at beamline $\mathrm{X} 22 \mathrm{C}$ at the National Synchrotron Light Source, and is part of a more detailed study involving both $\mathrm{La}_{0.7} \mathrm{Ca}_{0.3} \mathrm{MnO}_{3}$ and $\mathrm{Pr}_{0.7} \mathrm{Ca}_{0.3} \mathrm{MnO}_{3}$. ${ }^{16}$

The $\mathrm{La}_{0.7} \mathrm{Ca}_{0.3} \mathrm{MnO}_{3}$ single-crystal was grown using floating zone techniques at Bell Laboratories. The sample was determined to be fully twinned, with a $(110) /(002)$-oriented surface normal (in orthorhombic, Pbnm, notation), and mosaic width of $\sim 0.2$ degrees (FWHM). For simplicity, reflections are referenced using the (110) surface normal direction.

In a search for the presence of individual polarons, reciprocal space mesh scans-such as the one displayed below in Figure 1-were measured at temperatures both above and below the metal-insulator transition temperature $(\sim 252 \mathrm{~K})$. In contrast to recent neutron scattering results, ${ }^{14,15}$ there was no change in the intensity or shape of the $x$-ray diffuse scattering that would suggest the presence of individual polarons. Our measurements were carried out around the (220) Bragg peak using an incident X-ray energy of $6.535 \mathrm{keV}$ and a graphite analyzer, and it is possible that the scattering from individual polarons was below our detection limits. Another possibility compatible with our null result is that the diffuse scattering observed in the neutron scattering experiments is of magnetic origin.

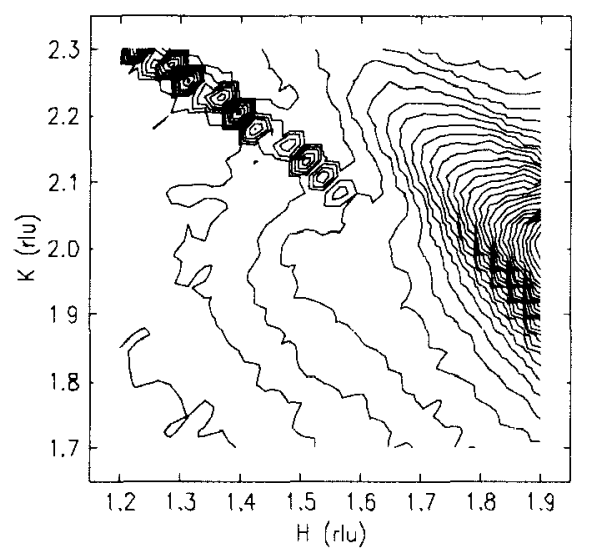

Figure 1. Reciprocal space mesh scan measured near the (220) Bragg peak, at a temperature of $260 \mathrm{~K}$. The arc of peaks between $\mathrm{H}=1.2, \mathrm{~K}=2.3$ and $\mathrm{H}=1.575, \mathrm{~K}=2.075$ is a powder line.

The second type of scattering-due to correlated polarons-was observed in the paramagnetic insulating phase of the sample. Specifically, diffuse peaks with ordering wavevectors of $(0.500)$ and $(00.50)$ were 


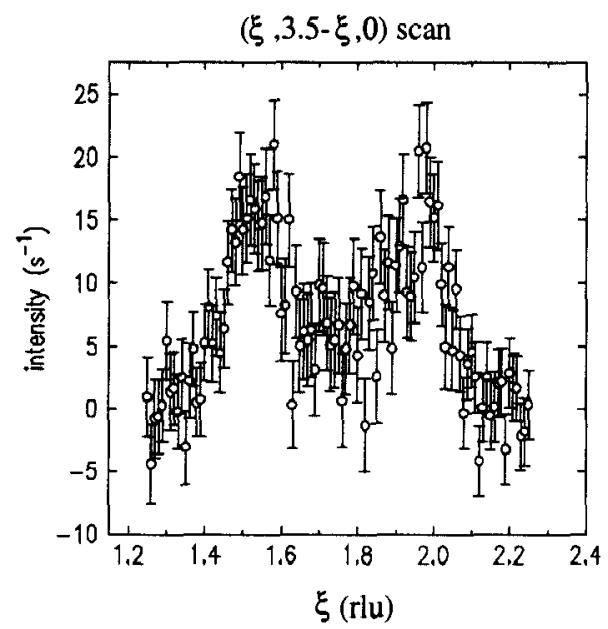

Figure 2. Difference between diagonal reciprocal space scans measured at temperatures of 260 and $220 \mathrm{~K}$.

observed, ${ }^{17}$ and found to decrease in intensity as the sample was cooled through the transition into the ferromagnetic metallic phase. Two of these well-resolved peaks, at (1.5 20 ) and (2 1.50$)$, are shown above in Figure 2.

The correlation length along the orthorhombic $a$ and $b$ directions was determined by using Lorentzian-squared fits to scans measured over the ( 1.520 ) peak, along both $\mathrm{H}$ and $\mathrm{K}^{18}$ Above the transition temperature, the correlation length was observed to be independent of temperature and isotropic, with a magnitude of 1-2 lattice constants.

The lack of diffuse scattering, the wavevector of the diffuse peaks, and the short correlation length suggest that the correlated polarons observed in the paramagnetic insulating phase of $\mathrm{La}_{0.7} \mathrm{Ca}_{0.3} \mathrm{MnO}_{3}$ are actually bipolarons, with a structure such as that displayed below in Figure 3. This bipolaron structure consists of two orthorhombic unit cells, with nextnearest-neighbor $\mathrm{Mn}^{4+}$ ions connected by orbitally-ordered $\mathrm{Mn}^{3+}$ ions. The structure can be viewed as either an orbital order domain in the CE-type phase or as a ferromagnetic zigzag, and we note that the lack of antiferromagnetic correlations reported by Adams et al. ${ }^{15}$ is consistent with the ferromagnetic nature of our bipolaron.

The presence of the diffuse peaks in the paramagnetic insulating phase emphasizes the importance of the role played by electron-phonon coupling in the $\mathrm{La}_{1-x} \mathrm{Ca}_{\mathbf{x}} \mathrm{MnO}_{3}$ system. Although long-range charge ordering has not been observed in this system for $\mathrm{x}<0.5$, strong electron-phonon coupling results in short-range correlations compatible with charge ordering at a doping as low as $x=0.3$. The high-temperature tendency toward ordering is only overcome at $T_{c}$, where the collapse of the bipolarons can be 


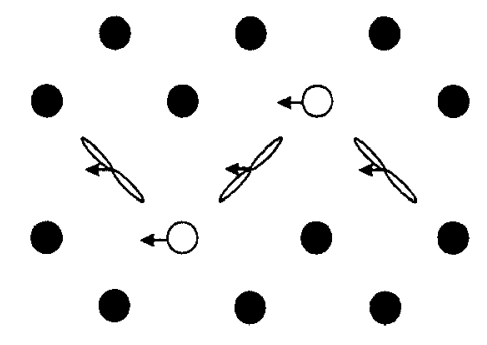

Figure 3. Schematic diagram of 2D ( $a-b$ plane) structure of CE-type bipolaron. Open

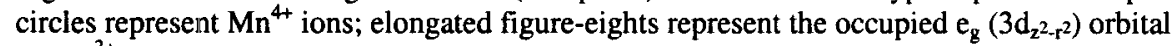
of $\mathrm{Mn}^{3+}$ ions; filled circles represent $\mathrm{Mn}$ ions that, on average, have the formal valence and no net orbital order; and arrows indicate the direction of the in-plane component of the magnetic moment.

attributed to the energy gain associated with itineracy in the ferromagnetic state.

\section{Acknowledgments}

The work at Brookhaven, both in the Physics Department and at the NSLS, was supported by the U.S. Department of Energy, Division of Materials Science, under Contract No. DE-AC02-98CH10886.

\section{References}

${ }^{1}$ C. Zener, Phys. Rev. 82, 403 (1951); P.W. Anderson and H. Hasegawa, Phys. Rev. 100, 675 (1955); P.G. deGennes, Phys. Rev. 118, 141 (1960).

${ }^{2}$ H. Roder, J. Zang, and A.R. Bishop, Phys. Rev. Lett. 76, 1356 (1996).

${ }^{3}$ A.J. Millis, B.I. Shraiman, and R. Mueller, Phys. Rev. Lett. 77, 175 (1996).

${ }^{4}$ M.F. Hundley et al., Appl. Phys. Lett. 67, 860 (1995).

${ }^{5}$ M. Jaime et al., Phys. Rev. Lett. 78, 951 (1997).

${ }^{6}$ S.J.L. Billinge et al., Phys. Rev. Lett. 77, 715 (1996).

${ }^{7}$ A. Shengalaya, G. Zhao, H. Keller, and K.A. Muller, Phys. Rev. Lett. 77, 5296 (1996).

${ }^{8}$ A.S. Alexandrov and A.M. Bratkovsky, Phys. Rev. Lett. 82, 141 (1999).

${ }^{9}$ K.H. Kim, M. Uehara, and S-W. Cheong, Phys. Rev. B 62, R1 1945 (2000).

${ }^{10}$ G.M. Zhao et al., Phys. Rev. B 62, R11949 (2000).

${ }^{11}$ S. Shimomura, N. Wakabayashi, H. Kuwahara, and Y. Tokura, Phys. Rev. Lett. 83, 4389 (1999).

${ }^{12}$ L. Vasiliu-Doloc et al., Phys. Rev. Lett. 83, 4393 (1999).

${ }^{13}$ S. Shimomura et al., Phys. Rev. B 62, 3875 (2000).

${ }^{14}$ P. Dai et al., Phys. Rev. Lett. 85, 2553 (2000).

${ }^{15}$ C.P. Adams et al., Phys. Rev. Lett. 85, 3954 (2000).

${ }^{16} \mathrm{C}$. S. Nelson et al., in preparation.

${ }^{17}$ Twinning of the sample and the width of the diffuse peaks make it impossible to determine whether or not there is a unique ordering wavevector.

${ }^{18}$ The correlation lengths are defined as $\xi_{\mathrm{a}} \equiv a / 2 \pi \Delta \mathrm{H}$ and $\xi_{\mathrm{b}} \equiv b / 2 \pi \Delta \mathrm{K}$, where $a$ and $b$ are the lattice constants and $\Delta H$ and $\Delta K$ are the HWHM values of the diffuse peaks along $H$ and $K$, respectively. 